\title{
Thalamic Stimulation Improves Postictal Cortical Arousal and Behavior
}

\author{
Jingwen Xu, ${ }^{1,6}$ Maria Milagros Galardi, ${ }^{1}$ Brian Pok, ${ }^{1}$ Kishan K. Patel, ${ }^{1}$ Charlie W. Zhao, ${ }^{1}$ John P. Andrews, ${ }^{1}$ \\ Shobhit Singla, ${ }^{1}$ Cian P. McCafferty, ${ }^{1}$ Li Feng, ${ }^{1}$ Eric. T. Musonza, ${ }^{1}$ Adam. J. Kundishora, ${ }^{1,3}$ \\ Abhijeet Gummadavelli, ${ }^{1,3}{ }^{\circledR}$ Jason L. Gerrard, ${ }^{3}{ }^{\circledR}$ Mark Laubach, ${ }^{4}$ Nicholas D. Schiff, ${ }^{5}$ and Hal Blumenfeld ${ }^{1,2,3}$ \\ ${ }^{1}$ Department of Neurology, Yale University School of Medicine, New Haven, Connecticut 06520, ${ }^{2}$ Department of Neuroscience, Yale University \\ School of Medicine, New Haven, Connecticut 06520, ${ }^{3}$ Department of Neurosurgery, Yale University School of Medicine, New Haven, Connecticut \\ 06520, ${ }^{4}$ Department of Biology, American University, Washington, DC 20016, ${ }^{5}$ Department of Neurology, Weill-Cornell Medical College, New York, \\ NY 10021, and ${ }^{6}$ Department of Neurology, Qilu Hospital, Cheeloo College of Medicine, Shandong University, Jinan, Shandong 250012, China
}

The postictal state following seizures is characterized by impaired consciousness and has a major negative impact on individuals with epilepsy. Previous work in disorders of consciousness including the postictal state suggests that bilateral deep brain stimulation (DBS) of the thalamic intralaminar central lateral nucleus (CL) may improve level of arousal. We tested the effects of postictal thalamic CL DBS in a rat model of secondarily generalized seizures elicited by electrical hippocampal stimulation. Thalamic CL DBS was delivered at $100 \mathrm{~Hz}$ during the postictal period in 21 female rats while measuring cortical electrophysiology and behavior. The postictal period was characterized by frontal cortical slow waves, like other states of depressed consciousness. In addition, rats exhibited severely impaired responses on two different behavioral tasks in the postictal state. Thalamic CL stimulation prevented postictal cortical slow wave activity but produced only modest behavioral improvement on a spontaneous licking sucrose reward task. We therefore also tested responses using a lever-press shock escape/avoidance (E/A) task. Rats achieved high success rates responding to the sound warning on the E/A task even during natural slow wave sleep but were severely impaired in the postictal state. Unlike the spontaneous licking task, thalamic CL DBS during the E/A task produced a marked improvement in behavior, with significant increases in lever-press shock avoidance with DBS compared with sham controls. These findings support the idea that DBS of subcortical arousal structures may be a novel therapeutic strategy benefitting patients with medically and surgically refractory epilepsy.

Key words: consciousness; deep brain stimulation; epilepsy; generalized tonic-clonic seizures; sleep; thalamus

Significance Statement

The postictal state following seizures is characterized by impaired consciousness and has a major negative impact on individuals with epilepsy. For the first time, we developed two behavioral tasks and demonstrate that bilateral deep brain stimulation (DBS) of the thalamic intralaminar central lateral nucleus (CL) decreased cortical slow wave activity and improved task performance in the postictal period. Because preclinical task performance studies are crucial to explore the effectiveness and safety of DBS treatment, our work is clinically relevant as it could support and help set the foundations for a human neurostimulation trial to improve postictal responsiveness in patients with medically and surgically refractory epilepsy.

Received May 31, 2020; revised Aug. 9, 2020; accepted Aug. 10, 2020.

Author contributions: J.X., C.P.M., M.L., N.D.S., and H.B. designed research; J.X., M.M.G., B.P., K.K.P., C.W.Z., and J.P.A. performed research; M.M.G., L.F., E.T.M., A.J.K., A.G., J.L.G., M.L., and N.D.S. contributed unpublished reagents/analytic tools; J.X., K.K.P., C.W.Z., J.P.A., S.S., C.P.M., E.T.M., and H.B. analyzed data; J.X., M.M.G., B.P., C.W.Z., S.S., C.P.M., L.F., E.T.M., A.J.K., A.G., J.L.G., M.L., N.D.S., and H.B. wrote the paper.

This work was supported by National Institutes of Health Grants R01 NS066974 and R01 NS096088, the Mark Loughridge and Michele Williams Foundation, the Betsy and Jonathan Blattmachr family (H.B.), and a China Scholarship Council fellowship (J.X.). We thank Dr. Kate Christison-Lagay for artwork in Figure $1 A$.

The authors declare no competing financial interests.

Correspondence should be addressed to Hal Blumenfeld at hal.blumenfeld@yale.edu.

https://doi.org/10.1523/JNEUROSCI.1370-20.2020

Copyright $(2020$ the authors

\section{Introduction}

The postictal state that follows epileptic seizures comprises a broad spectrum of disturbances including behavior, motor function, cognitive, autonomic, and psychological changes and can last from minutes to days (Helmstaedter et al., 1994; Fisher and Schachter, 2000; Todd, 2005; Rémi and Noachtar, 2010; Schmidt and Noachtar, 2010; Theodore, 2010; Werhahn, 2010; Carlsson et al., 2011). Postictal symptoms can be more debilitating for patients with epilepsy than the ictal events. It has been shown that postictal cognitive and behavioral impairment have a significant correlation with depression in adults with epilepsy (Josephson et al., 2016). Postictal consciousness impairment in 
school-aged children is a cause of decreased attention that negatively impacts their education (Elliott et al., 2005). Postictal phenomena prevented over $50 \%$ of affected children from returning to their normal activities of childhood (MacEachern et al., 2017). Furthermore, longer postictal generalized EEG suppression duration has been associated with risk of sudden unexpected death in epilepsy (SUDEP; Hesdorffer and Tomson, 2013; Seyal et al., 2013; Rugg-Gunn et al., 2016; Tomson et al., 2016). Importantly, postictal immobility duration is associated with respiratory dysfunction (Kuo et al., 2016), which may be a causal factor in SUDEP (Ryvlin et al., 2013; Zhan et al., 2016). Given all of these findings, reversing impaired arousal and postictal immobility may reduce SUDEP risk and could significantly improve the quality of life of people with epilepsy.

In recent years, neurostimulation of subcortical regions has emerged as a promising therapy for the treatment of refractory seizures (Fisher and Velasco, 2014; Heck et al., 2014; BenedettiIsaac et al., 2015; Klinger and Mittal, 2018). The mechanism by which consciousness is lost during and after temporal lobe seizures has been consolidated in the "network inhibition hypothesis;" which posits that inhibition of subcortical arousal structures may result in cortical states resembling deep sleep or coma during and after seizures (Norden and Blumenfeld, 2002; Blumenfeld, 2012). Thus, therapeutic activation of these subcortical arousal structures could attenuate the loss of consciousness experienced in focal seizures.

Specifically, the intralaminar thalamic nuclei receive input from the brainstem arousal system and have been shown to play an important role in awareness (Van der Werf et al., 2002; Schiff et al., 2007, 2013; Baker et al., 2016). These nuclei contain matrix neurons that project to the cortex and synchronize activity in neural networks that drive cognitive functions (Jones, 2009). Previous studies have demonstrated benefits of stimulating the thalamic intralaminar central lateral nucleus (CL) in restoring arousal. In asleep rats, central thalamic stimulation produced widespread activation of the forebrain (Liu et al., 2015). Deep brain stimulation (DBS) within CL and the medial dorsal thalamic tegmental tract in nonhuman primates produced a robust modulation of performance and arousal, (Baker et al., 2016). In a landmark human study, thalamic CL DBS improved arousal and functional behavioral performance for a patient in the minimally conscious state (Schiff et al., 2007). More recently, CL stimulation was found to effectively restore arousal and wake-like neural processing in anesthetized macaques (Redinbaugh et al., 2020). Previous studies in a rodent model of limbic seizures have also shown that bilateral CL DBS improved cortical electrophysiological arousal and spontaneous locomotor behaviors during deep anesthesia, sleep, and the postictal period (Gummadavelli et al., 2015a; Kundishora et al., 2017). However, whether purposeful goal-oriented behavior can be restored in the postical period through CL DBS remains to be determined and is crucial for translational value to human therapy.

Here, our aim is to further investigate CL DBS effects in the postictal period using a rodent model of secondarily generalized limbic seizures. We tested two different behavioral tasks for the first time in an animal model of postictal unconsciousness; a positive reinforcement spontaneous-licking sucrose reward task and a negative reinforcement lever-press escape-avoidance task. By assessing electrophysiological changes and behavioral performance in these tasks, we provide evidence that therapeutic neurostimulation in thalamic CL can reduce cortical slow-wave activity and improve behavioral task performance in the postictal period.

\section{Materials and Methods}

Animals

All procedures were approved by the Yale University Institutional Animal Care and Use Committee. Healthy adult female Sprague Dawley rats weighing 180-250 g (Charles River Laboratories) were used. We used female animals because prior studies in our lab established a reliable awake stimulation-based seizure model with females originally chosen because of their smaller size facilitating functional magnetic resonance imaging (fMRI) studies in the same model (Gummadavelli et al., 2015a; Motelow et al., 2015; Kundishora et al., 2017).

\section{Behavioral apparatus and tasks}

All components of the behavioral set-up, unless otherwise specified, were obtained from Med Associates. Two model ENV-008-CT operant chambers were used (Fig. 1A). They measured $29.53 \times 24.84 \times$ $18.67 \mathrm{~cm}$ (length $\times$ width $\times$ height) and were individually placed within model ENV-018MD-EMS sound-attenuating chambers with electronic shielding.

Sucrose reward/spontaneous licking task

For the sucrose reward/spontaneous licking task, one wall of the chamber was fitted with a photobeam lickometer with a sipper tube for infusion delivery (CT-ENV-251L-X4). The sipper tube was connected via plastic tubing to a syringe operated by a single-speed syringe pump (PHM-100, speed 3.33 RPM) that was calibrated by adjusting the duration of pump activation ( 3 s) to deliver $90 \mu \mathrm{l}$ of $20 \%$ sucrose solution when activated. On the opposite wall of the modular chamber, a houselight (ENV-215 M) was constantly lit during experiments for general illumination. On one wall of the sound attenuating chamber, a fan provided constant background noise and ventilation. The house-light and fan were turned on at the beginning of the behavioral sessions and remained on until the end. Different components of the behavioral apparatus were interfaced using a SmartCtrl Connection Panel (DIG-716). Data were collected using Med-PC software (Med Associates). Chambers were cleaned following each session.

For the sucrose reward spontaneous licking task, rats were trained to collect liquid sucrose from the sipper tube in the modular chamber. After acclimating to their living conditions, rats were food restricted to $36 \mathrm{~g} / \mathrm{kg}$ of lab chow per day. When animals reached $\sim 90 \%$ of their initial weights, $30 \mathrm{ml}$ of $20 \%$ sucrose solution was offered in a bottle during a 30-min period per day for them to become familiar with the taste. After $3 \mathrm{~d}$ of sucrose water exposure, training in the behavioral chamber started (Fig. 1B). Each trial within the training session began with a 10 -s period of sucrose-availability window during which the rat could obtain $90-\mu \mathrm{l}$ sucrose solution if they licked the spout. After the rat received the sucrose solution, which was considered a "reward," or after the 10-s period ended, a random delay period of sucrose reward unavailability would start. Duration of the delay was chosen randomly from $10,15,20,25$, or $30 \mathrm{~s}$. Each trial ended after the end of this delay period and a new trial would begin (Fig. 1B). The daily session would end after the rat received 90 rewards or after $45 \mathrm{~min}$. Only one session was done each day during training.

When a rat received $\geq 90 \%$ rewards of those offered on at least $2 \mathrm{~d}$ in three successive days (and the total training days were at least $4 \mathrm{~d}$ ), surgical electrode implantation and initial stimulus titration was performed (Fig. 1D). After a one-week recovery period from electrode implantation, rats were retrained on the same task to receive $\geq 90 \%$ rewards. Stimuli were then again titrated in the awake state (see below, Stimulus titration and electrophysiology/video recording procedures), and finally testing during seizures began (Fig. $1 B, D$ ). Of the 15 rats initially successfully trained, three rats were excluded and euthanized because of surgical complications, resulting in a final sample size in the sucrose reward/ spontaneous licking experiments of 12 rats.

Two testing sessions were conducted each day, with a minimum 3-h delay between the two sessions. During each testing session, after the rat received a total of 30 rewards, current was delivered to the hippocampus (HC; for all stimulation parameters, see below, Stimulus titration and electrophysiology/video recording procedures) to trigger a secondarily 
A

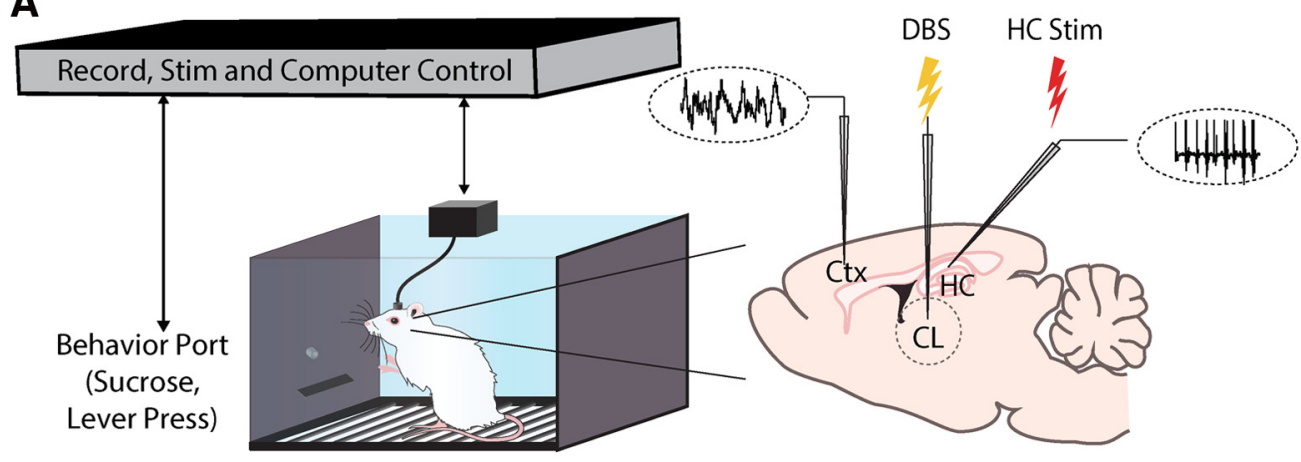

B Sucrose Reward/ Spontaneous Licking Task

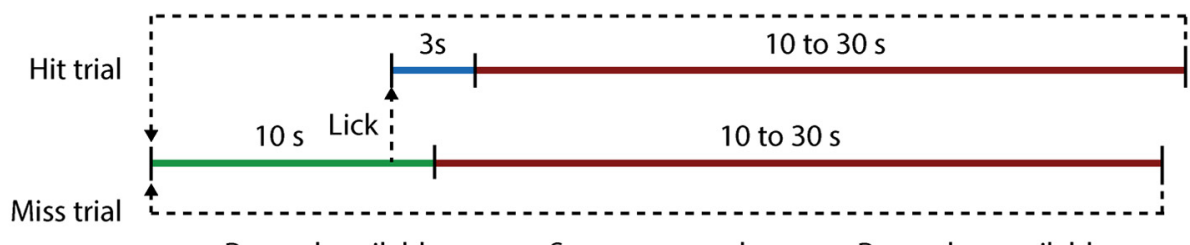

C Escape/Avoidance (E/A) Task

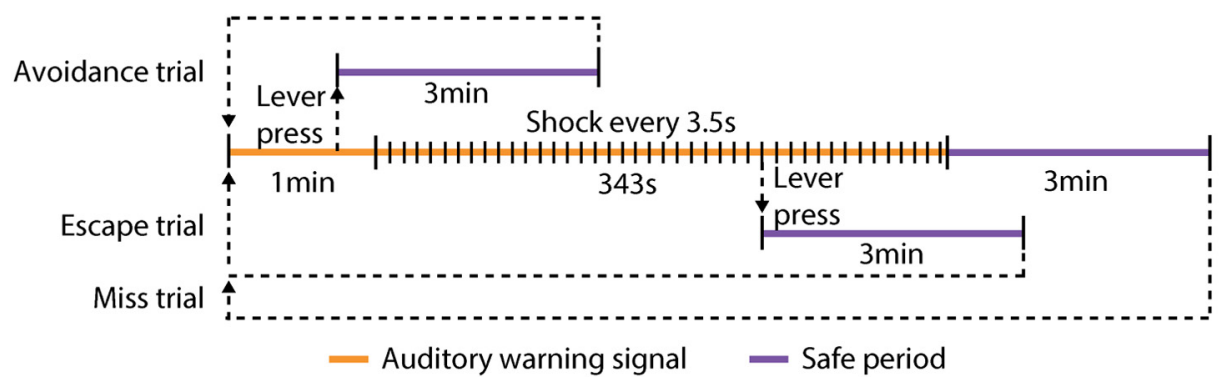

D Experimental protocol

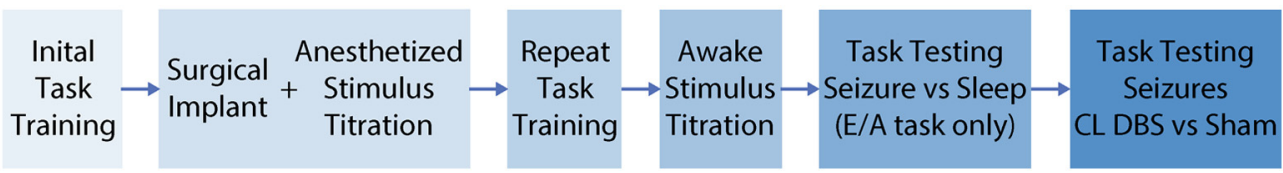

Figure 1. Experimental setup and design. $\boldsymbol{A}$, Behavioral testing and recording setup. $\boldsymbol{B}$, Sucrose reward/spontaneous licking task timeline. $\boldsymbol{C}$, E/A task timeline. $\boldsymbol{D}, 0$ verall experimental protocol for both the spontaneous licking and E/A tasks. Ctx, frontal cortex.

generalized seizure, defined as polyspike activity in both $\mathrm{HC}$ and frontal cortical local field potential (LFP) and behavioral Racine class 5. A 600-s bilateral central lateral thalamic stimulation or sham stimulation was initiated simultaneously (Fig. 2A) to test the effects of DBS on cortical physiology and on task performance on the sucrose reward spontaneous licking task during the ictal and postictal periods (Fig. 3). A testing session ended after the rat received a total of 90 rewards or after $45 \mathrm{~min}$. Two sessions, with at least a 3-h interval between sessions, were performed each day with one seizure per session, and alternating thalamic CL DBS and sham DBS stimulation. First stimulus type (sham or DBS) was counterbalanced across days. Testing was continued for an average of 9.2 sessions ( $4.6 \mathrm{~d}$ ) per animal (range 6-10 sessions).

Lever-press escape/avoidance (E/A) task

Training was conducted in the same behavioral chamber set-up as the sucrose reward task (Fig. 1A) with the modifications described below in this section. The chamber wall that was opposite to the house light (always on) was fitted with a lever (ENV-110 M, $10.5 \mathrm{~cm}$ above the floor), a stimulus light (ENV-221 M, $20.5 \mathrm{~cm}$ above the floor), and a speaker (ENV-224AM, $26 \mathrm{~cm}$ above the grid floor). A $1.0 \mathrm{~mA}, 0.5-\mathrm{s}$ electric foot-shock was delivered through the grid floor as an aversion stimulation (ENV-414S-ASRP). The warning signal was a $75-\mathrm{dB}$ white noise. Safe periods were indicated by the stimulus light alternately flashing on for $1 \mathrm{~s}$ and off for $1 \mathrm{~s}$.

E/A training was performed as described previously by Servatius et al. (2008). Sessions consisted of 20 trials. One session was performed per day during training. A trial commenced with the delivery of the auditory warning signal (Fig. 1C). In the first three training sessions, onset of the auditory warning signal coincided with onset of the foot shock stimulus delivered every $3.5 \mathrm{~s}$. Subsequently, from session four onward a 1-min warning period was introduced without shock delivery (Fig. 1C). A lever press during the first $60 \mathrm{~s}$ of the warning signal period prevented the 
A

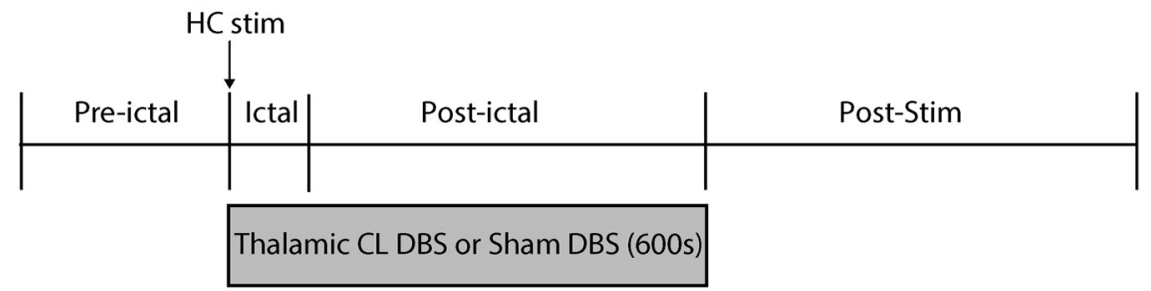

B

Pre-ictal

Ictal

Post-ictal

Post-Stim

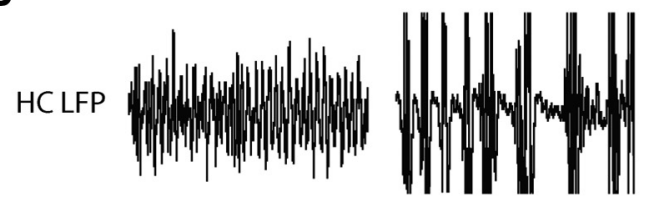

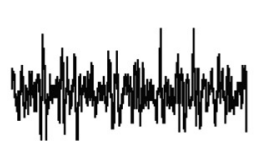

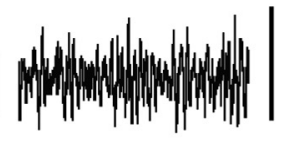

$200 \mu \mathrm{V}$

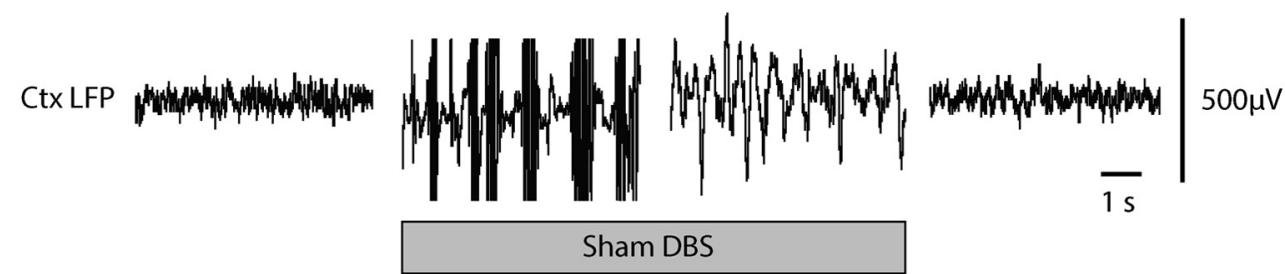

C
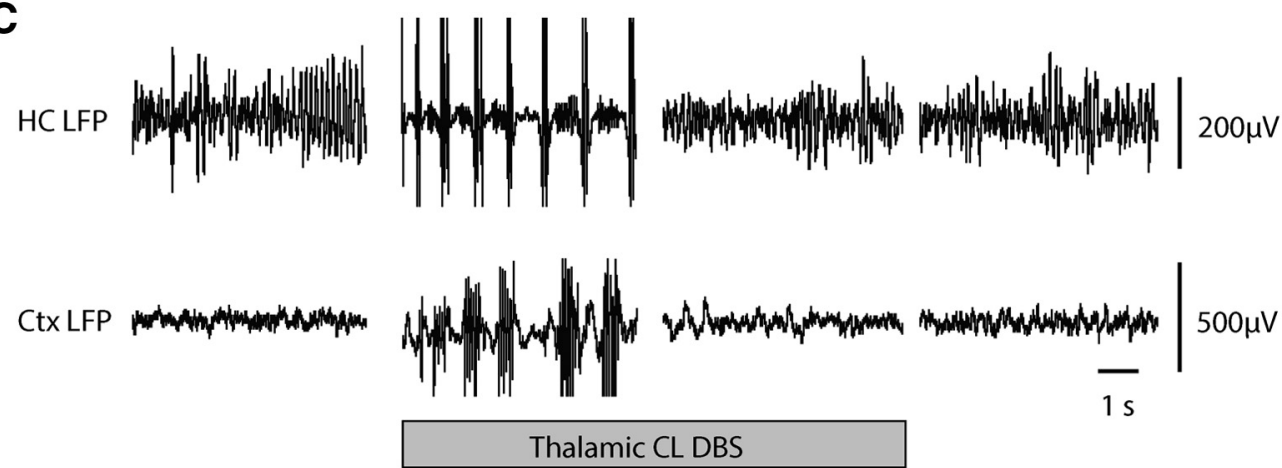

Figure 2. Thalamic CL DBS produces cortical physiological arousal in the postictal period during the sucrose reward spontaneous licking task. $\boldsymbol{A}$, Experimental protocol and sequence of preictal, ictal, postictal, and poststimulation (post-stim) epochs. $\mathrm{HC}$ stimulation for $2 \mathrm{~s}$ at $60 \mathrm{~Hz}$ induces a secondarily generalized seizure. Simultaneous with seizure onset, bilateral thalamic CL DBS or sham control DBS is initiated and continued for $5 \mathrm{~min}(600 \mathrm{~s})$ total, typically spanning both the ictal and postictal periods. $\boldsymbol{B}$, Recordings during sham DBS. Preictal, ictal, postictal, and poststim time samples exemplify secondarily generalized seizures evidenced by polyspike discharges in both HC and orbital frontal Ctx LFP during the ictal period; prominent slow waves in Ctx LFP during the postictal period; and recovery in the post-stim period. C, Recordings during therapeutic thalamic CL DBS $(100 \mathrm{~Hz}, 100-\mu \mathrm{A}$ split approximately equally between the two thalami). Thalamic CL DBS markedly decreases postictal slow wave activity in Ctx compared with sham control (B). Ctx, orbital frontal cortex.

delivery of a shock, turned off the auditory warning signal, triggered a 3min safe period signaled by a flashing light, and was scored as an "avoidance." A lever press during the warning signal period, but after $60 \mathrm{~s}$ elapsed, terminated shock delivery, turned off the auditory warning signal, triggered a 3-min safe period signaled by a flashing light, and was scored as an "escape." In the absence of a lever press, a maximum of 99 0.5 -s shocks were delivered on a fixed 3.5-s interval schedule leading to the automatic end of a trial and beginning of the safe period, again signaled by ending the auditory warning signal and by a flashing light. The next trial began after the end of the safe period (Fig. 1C). A rat that failed to have a lever-press response by the end of the fourth training session was removed from the study. This occurred in 12 of 32 rats tested.

When a rat achieved $\geq 90 \%$ avoidance of the 20 trials in each session over two successive days on the E/A task (and the total training time was at least $6 \mathrm{~d}$ ), surgical electrode implantation and initial stimulus titration was performed (Fig. 1D). Four additional rats were excluded at this stage because of surgical complications. After a one-week recovery period from electrode implantation, rats were retrained to achieve $\geq 90 \%$ avoidance. Five out of 16 rats were excluded for failing to achieve $\geq 90 \%$ avoidance within three sessions after surgery. Stimuli were again titrated in the awake state (see below, Stimulus titration and electrophysiology/ video recording procedures) and finally E/A task testing began during seizures and during naturally occurring sleep (Fig. 1C,D). Two more rats were excluded because of electrodes not falling in target regions on histologic review (see below, Histology) resulting in a final sample size for the $\mathrm{E} / \mathrm{A}$ experiments of nine rats.

To compare E/A task performance in naturally occurring sleep to the postictal period, we performed sleep testing before seizure testing in the same session. Animals were housed with lights off at 7 P.M. and on at 7 A.M., and sessions began at 8-9 A.M. each day when natural sleep was common. Only one sleep versus seizure session was done each day. In a sleep versus seizure testing session, we would first allow the rats to attain up to five avoidances using the usual 3-min safe period between trials (Fig. 1C). We then extended the safe period typically up to $15-30 \mathrm{~min}$ to allow the animal to spontaneously fall asleep based on sleep-related cortical slow waves observed continuously for $\geq 10 \mathrm{~s}$. Some animals fell 

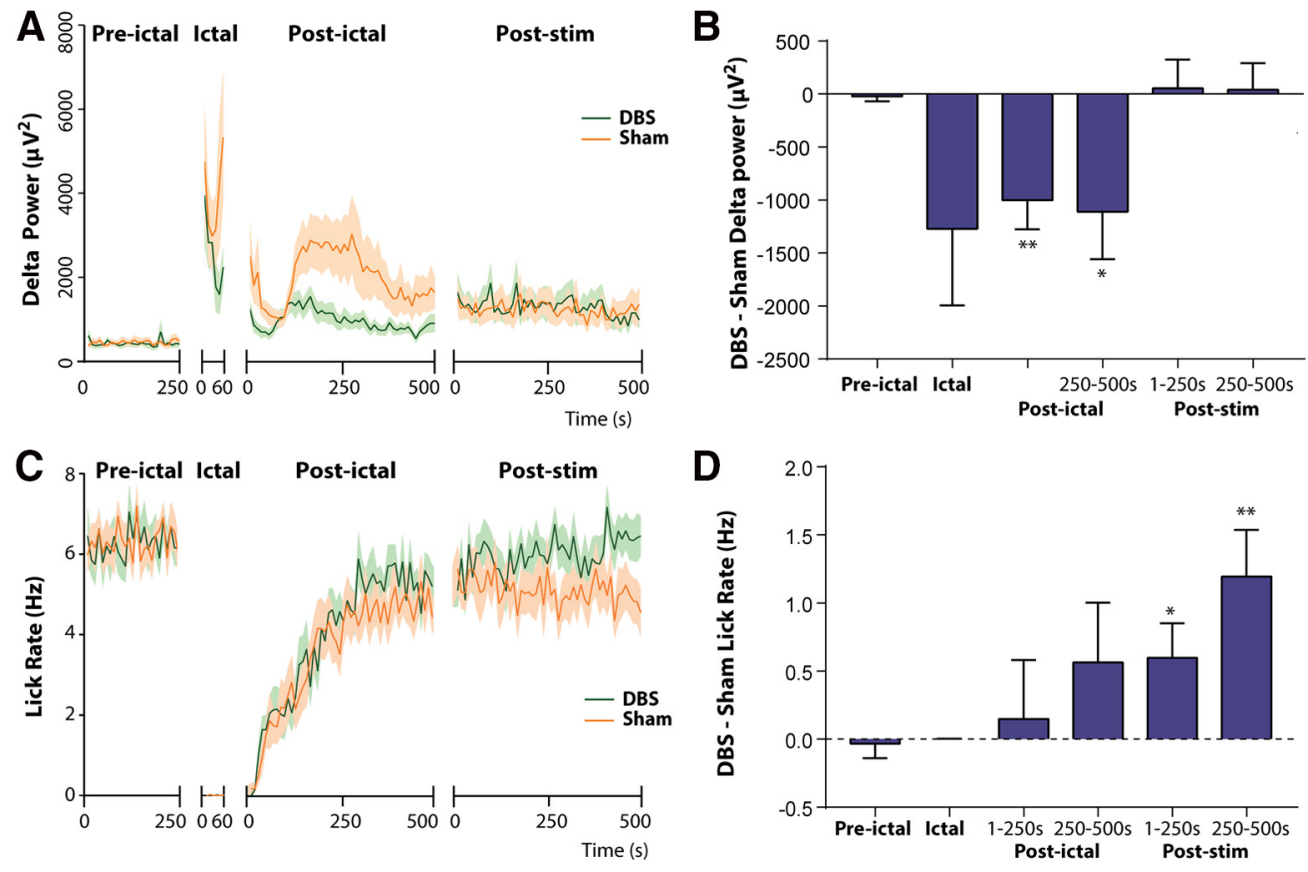

Figure 3. Effects of bilateral thalamic $\mathrm{CL}$ stimulation on postictal cortical slowing and sucrose reward spontaneous licking task performance. $A$, Time course of delta-band (0-4 Hz) orbital frontal cortex LFP power in DBS group compared with sham group during preictal, ictal, postictal, and post-stim epochs. B, Delta-Band power in DBS minus sham controls is significantly reduced during the ictal, postictal 1- to 250-s, and postictal 250- to 500-s epochs. C, Time course of licking rate in sucrose reward task throughout different epochs. Spontaneous licking is dramatically reduced in the ictal period and then gradually recovers. $\boldsymbol{D}$, Licking rate in DBS minus sham controls gradually increases in the postictal period and reaches statistical significance during the post-stim 1- to 250-s and post-stim 250- to 500-s epochs; $n=12$ animals, 55 sham sessions, 55 DBS sessions. All results are mean \pm SEM. The first $60 \mathrm{~s}$ of seizure activity were used for analysis of the ictal epochs. Values in A and C were calculated in 10-s non-overlapping epochs; ${ }^{*} p<0.05,{ }^{* *} p<0.01$.

asleep during the safe periods even before five avoidances, and these animals were also entered into sleeping E/A trials once $\geq 10$-s continuous cortical slow waves were observed. Sleeping E/A task trials were then initiated by the warning auditory signal (Fig. 1C). Up to five sleeping E/A trials were performed in each session unless the rat did not fall asleep within $1 \mathrm{~h}$. After the sleeping trials, the rats next performed at least three awake E/A trials with the usual 3-min safe period, which were followed by the seizure trial. At the end of the safe window, current was delivered to the HC (see below, Stimulus titration and electrophysiology/video recording procedures) to trigger a secondarily generalized seizure. Like in the sleep trials, a new E/A task trial would start in the postictal period signaled by the warning auditory signal after cortical slow waves were observed continuously for $\geq 10 \mathrm{~s}$ after the seizure. The session would end after the seizure trial. Sleep versus seizure E/A testing was continued for an average of 2.0 sessions ( $2.0 \mathrm{~d}$ ) per animal (range 1-3) (Fig. 4).

$\mathrm{E} / \mathrm{A}$ testing was next done during seizures to investigate effects of thalamic CL DBS (Fig. 1D). During a testing session, after the rat achieved a total of five avoidances, current was delivered to the HC (see below, Stimulus titration and electrophysiology/video recording procedures) to trigger a secondarily generalized seizure. Unlike the spontaneous licking task where behavior readout was monitored continuously, the E/A task yielded a single response measurement once every few minutes (Fig. 1C). Therefore, we targeted both the E/A behavioral testing and DBS treatment to the time of maximal physiological impairment in the postictal period, which typically occurred several minutes after seizures (see Fig. $3 A$, postictal delta power time course). Thus, a 120-s bilateral CL stimulation or sham stimulation started after $10 \mathrm{~s}$ of continuous electrographic slow waves in the postictal period, and a new E/A trial (warning sound on) would begin $10 \mathrm{~s}$ after the CL stimulation/sham stimulation onset (Fig. 5A). Each E/A testing session ended after the DBS (or sham) trial was complete. Two sessions, with at least a 3-h interval between them, were performed each day, alternating thalamic CL DBS and sham stimulations. First stimulus of the day (DBS or sham) was counterbalanced across days. Seizure testing with the E/A task was continued for an average of 10.9 sessions (5.4 d per animal (range 9-12 sessions).

\section{Surgery and electrode implantation}

The animal model of focal limbic seizures with secondary generalization and electrode implantation was prepared as described in detail previously (Gummadavelli et al., 2015a), summarized briefly below in this section. Animals were deeply anesthetized with ketamine and xylazine. Craniotomies were done stereotaxically over sites of planned electrode placement, anchoring screws and ground screw, with all coordinates reported as final electrode tip locations in reference to bregma (Paxinos and Waston, 1998). A twisted pair bipolar electrode was implanted into the right lateral orbitofrontal cortex (AP $+4.2 \mathrm{~mm}$; $\mathrm{ML}+2.2 \mathrm{~mm}$; SI $-4.2 \mathrm{~mm}$ ). Two twisted pair bipolar electrodes were implanted in bilateral CLs (AP $-2.8 \mathrm{~mm}$; ML $\pm 1.4 \mathrm{~mm}$; SI $-5.4 \mathrm{~mm}$ ). Two twisted pair bipolar electrodes were implanted in the bilateral dorsal HC (AP -3.8 $\mathrm{mm}$; $\mathrm{ML} \pm 2.5 \mathrm{~mm}$; SI $-3.6 \mathrm{~mm}$ ). Electrodes were firmly cemented to nearby anchoring screws and placed into two six-pin pedestals (MS363; PlasticsOne). Chronic recordings began at least oneweek postoperatively.

\section{Stimulus titration and electrophysiology/video recording procedures}

We undertook electrophysiological recordings under the following conditions (Fig. 1D): (1) acute recordings were performed under anesthesia during electrode implantation to confirm electrode locations and for initial titration of stimulus amplitudes; (2) recordings in chronically implanted animals were performed in behavioral chambers in the awake state or during naturally occurring sleep for final stimulus titration and for experimental task testing.

Stimulations of CL and $\mathrm{HC}$ were generated by independent isolated pulse stimulators (Model 2100, A-M Systems). Stimulus current amplitudes were titrated individually per animal, first acutely under anesthesia and then in chronic recordings before task testing (Fig. 1D).

For CL stimulation, stimulus frequency was chosen based on prior work (Schiff et al., 2007; Gummadavelli et al., 2015a; Kundishora et al., 2017) demonstrating that $\sim 100 \mathrm{~Hz}$ was most effective in CL to produce cortical physiological arousal. We therefore used a $100-\mathrm{Hz}$ biphasic square wave $(0.5 \mathrm{~ms}$ per phase) stimulus train in CL. For HC stimulation, to induce focal limbic seizures with secondary generalization as in 
prior work (Englot et al., 2008; Gummadavelli et al., 2015a; Zhan et al., 2016) we used a 2 s, $60-\mathrm{Hz}$ biphasic square wave ( $1 \mathrm{~ms}$ per phase) hippocampal stimulus.

Stimulus current amplitudes were first titrated under anesthesia during acute recordings at the time of electrode implantation (Fig. 1D). We started by electrically stimulating bilateral CL under deep anesthesia (defined as continuous cortical slow wave activity) to determine stimulus current and electrode placement effective in producing cortical physiological arousal as measured by decreased slow waves. The stimulus current was split between the bilateral CL electrodes, beginning at $100 \mu \mathrm{A}$ with 20 -s train duration, and was titrated upwards in $100-\mu \mathrm{A}$ increments until cortical physiological arousal was achieved. Final effective current amplitude ranged from 100 to $500 \mu \mathrm{A}$ split bilaterally ( $\sim 50-250 \mu \mathrm{A}$ per side). Higher amperages during titration were tested as well to ensure a lack of after-discharges or seizure induction from the CL stimulation.

We next titrated HC stimulus amplitude during light anesthesia, defined as when animals had recovered from deep anesthesia to a state in which less than three cortical slow waves occurred per $10 \mathrm{~s}$, but animals remained unresponsive to foot pinch as described previously (Englot et al., 2008). This light anesthesia state enabled us to see the emergence of postictal slow waves from a baseline which had very little slow wave activity (predominance of cortical fast activity). Seizures were induced with a 2 -s unilateral HC stimulus (parameters above). Stimulus current began at $100 \mu \mathrm{A}$ and was titrated upward (increments 50$100 \mu \mathrm{A}$ ) to induce a secondarily generalized seizure based on polyspike activity in both HC and frontal cortical LFP, followed by a postictal period with cortical slow wave activity. Final HC stimulus current for individual animals ranged from $100 \mu \mathrm{A}$ to $1.5 \mathrm{~mA}$.

We next again titrated CL stimulus amplitude under light anesthesia in the postictal period. After seizure termination, postictal cortical slow waves were observed. Bilateral CL were stimulated with a 20 -s train as above, beginning at the current that previously produced effective cortical physiological arousal under anesthesia, and titrating upward. Final bilateral CL stimulus amplitude again ranged from 100 to $500 \mu \mathrm{A}(\sim 50-$ $250 \mu \mathrm{A}$ per side), titrated individually per animal, to obtain cortical physiological arousal as measured by decreased cortical slow waves in the postictal period.

Following electrode implantation, a secondary stimulus titration was performed in unanesthetized animals at least one week after recovery from surgery and before behavioral tasks (Fig. 1D). Stimulus titration was first performed under natural sleep, defined as cortical slow waves for at least $20 \mathrm{~s}$ of each 30 -s recording epoch. After a baseline of at least $10 \mathrm{~s}$ of continuous slow waves, bilateral CL were stimulated with a 20 -s train to produce decreased cortical slow waves and behavioral arousal. Final range of CL stimulation delivered bilaterally in all experiments was $100-500 \mu \mathrm{A}(\sim 50-250 \mu \mathrm{A}$ per side). We next again titrated HC stimulus amplitude in chronically implanted animals to obtain secondarily generalized seizures (polyspike activity in both $\mathrm{HC}$ and frontal cortical LFP and behavioral Racine class 5) and to effectively evoke a postictal period with impaired behavior and cortical slow waves. HC current was greater (typically $>2 \mathrm{~mA}$ ) to obtain secondarily generalized seizures in awake animals. Finally, we titrated postictal CL stimulus amplitude again, the same way as in sleep. Final range of chronic bilateral CL stimulation was $100-500 \mu \mathrm{A}(\sim 50-250 \mu \mathrm{A}$ per side) to produce cortical physiological and behavioral arousal. The current amplitudes thus obtained for sleep and the postictal state were recorded separately for each individual animal and were then used in all subsequent behavioral task testing as described in the sections above (Fig. 1D). Sham control stimulation of CL was performed with $0.2-\mu \mathrm{A}$ split bilaterally $(\sim 0.1 \mu \mathrm{A}$ per side) in all animals. Note that although 20 -s CL stimulation trains were used during titration, longer duration trains were used during tasks (600 s for the spontaneous licking sucrose reward task; $120 \mathrm{~s}$ for the E/A task).

Electrophysiological recordings of cortical and $\mathrm{HC}$ signals were acquired on separate channels of microelectrode amplifiers (A-M Systems Model 1800). In acute recordings, LFP signals were recorded with $1000 \times$ amplifier gain and filtered at $1-500 \mathrm{~Hz}$. In chronically implanted animals, two six-pin connector wires (PlasticsOne) were connected to a single 12-pin commutator (PlasticOne). The commutator was then connected to the head stages of the A-M Systems amplifiers using two customized six-pin connectors (363SL-6; PlasticsOne). HC and cortical LFP signals were filtered at $1-500 \mathrm{~Hz}$ on the recording amplifiers with $1000 \times$ gain.

All signals in both acute and chronic experiments were digitized with an analog-to-digital converter (Power 1401; CED) using Spike2 (version 5.2; CED). Cortical and HC LFP were sampled at $1000 \mathrm{~Hz}$. The output BNC breakout box (SG-726-TTL) of the behavioral chamber was connected to a digital input BNC breakout box (2805DI-8) from CED, which further sent the behavior data to the digital input socket of CED Power 1401 to synchronize behavioral data with electrophysiological recording. The behavior events were shown as event marks in Spike2. Video was captured with a digital camera $\left(180^{\circ}\right.$ Fisheye Lens 1080 pWide Angle Pc Web USB Camera, Ailipu Technology Co, Ltd) and recorded with Spike2 Video Recorder (CED).

\section{Histology}

After completing behavioral and electrophysiological recordings, we performed histology to confirm all electrode locations as described in our previous work (Gummadavelli et al., 2015a; Feng et al., 2017; Kundishora et al., 2017). Briefly, animals were euthanized and perfused, brains were removed and postfixed, and $100-\mu \mathrm{m}$ slices were then stained with cresyl violet. Electrode locations for the central lateral thalamus were confirmed using the following landmarks (Paxinos and Waston, 1998): location ventral to the HC in the dorsal thalamus and just lateral to the stria medullaris/habenula complex as described in our previous work (Gummadavelli et al., 2015a; Feng et al., 2017; Kundishora et al., 2017). Any animals with incorrect electrode locations were excluded from analysis.

\section{Data analysis and statistics}

Electrophysiological data were analyzed in Spike2 (CED) and Microsoft Excel (Microsoft). Analysis epochs were defined as follows:

1. For time-course of sucrose reward task (Figs. 2, 3), the preictal epoch was defined as the $250 \mathrm{~s}$ immediately preceding hippocampal stimulation; ictal was defined as the $60 \mathrm{~s}$ immediately following hippocampal stimulation; postictal was defined as the $500 \mathrm{~s}$ immediately following end of seizure, during CL stimulation; poststimulation (post-stim) was defined as the $500 \mathrm{~s}$ immediately following the termination of CL stimulation.

2. For experiments comparing the postictal period to naturally occurring sleep in the lever-press E/A task (Fig. 4), awake was defined as the $10 \mathrm{~s}$ before appearance of consistent sleep slow waves; presound was defined as the $10 \mathrm{~s}$ immediately preceding sound onset; postsound was defined as the $10 \mathrm{~s}$ immediately following sound onset. The preictal period was defined as $10 \mathrm{~s}$ immediately preceding hippocampal stimulation, postictal presound was defined as $10 \mathrm{~s} \mathrm{im-}$ mediately preceding sound onset, after $10 \mathrm{~s}$ of continuous electrographic slow waves in the postictal period, and postictal postsound was defined as $10 \mathrm{~s}$ immediately following sound onset during the postictal period.

3. For experiments in the postictal period with the lever-press E/A task comparing CL DBS vs sham (Figs. 5, 6), sham or DBS preictal baseline was defined as the $10 \mathrm{~s}$ immediately preceding hippocampal stimulation; sham or DBS postictal pre-stim was defined as the $10 \mathrm{~s}$ immediately preceding CL stimulation; sham or DBS postictal stim was defined as the first $10 \mathrm{~s}$ immediately following CL stimulation.

For each epoch, power was measured from the cortical LFP (FFT size in Spike-2 was $1.024 \mathrm{~s}$, Hanning window, yielding a frequency resolution of $\sim 1 \mathrm{~Hz}$ ).

Behavioral data were exported from Med-PC acquisition software for analysis in MATLAB (The MathWorks), Python (The Python Software Foundation), and Microsoft Excel. During the sucrose reward task, licking rate was defined as the number of spout licks per second. During the lever-press E/A task, avoidance percentage was defined as the ratio between avoidance trials and total trials of any particular trial type; response time was defined as the period from sound onset to lever press.

Trials were excluded from analysis based on the following criteria: (1) seizure elicited was focal without polyspike discharges in frontal 
A

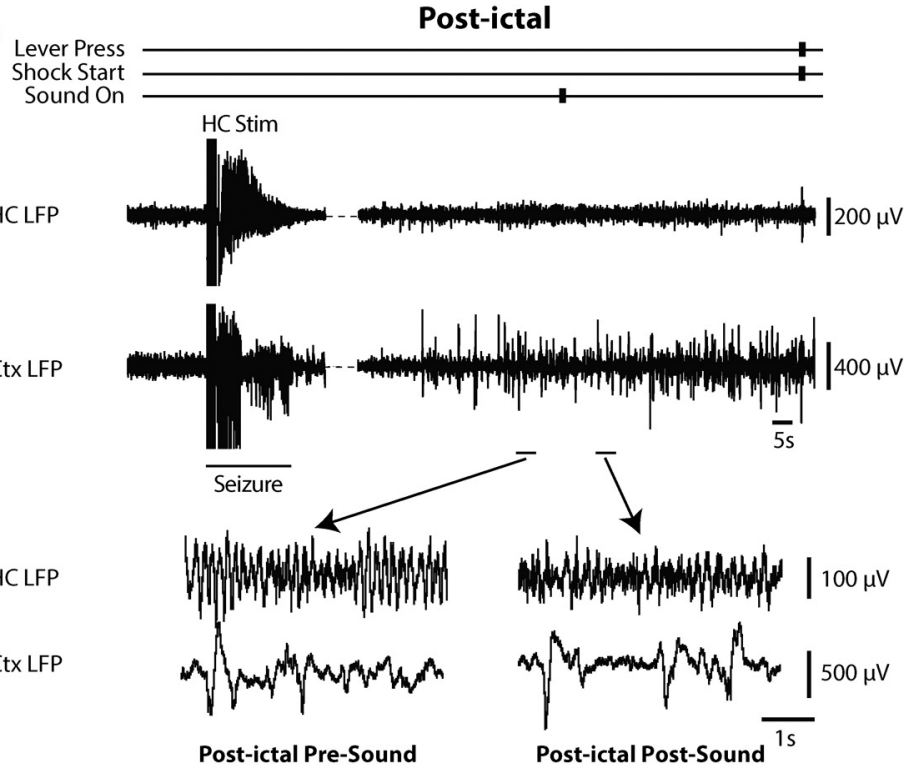

B

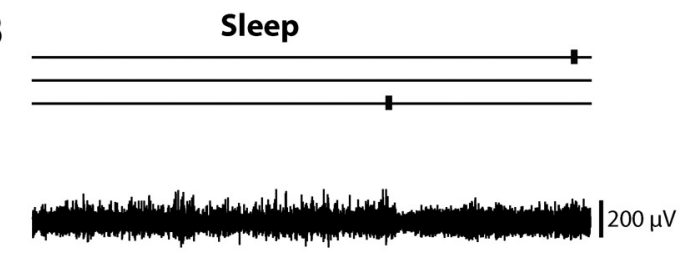

C

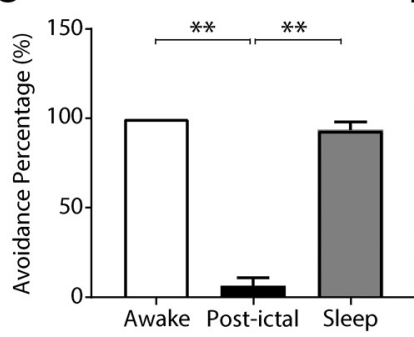

D

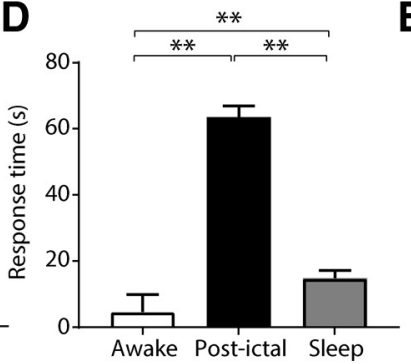

E

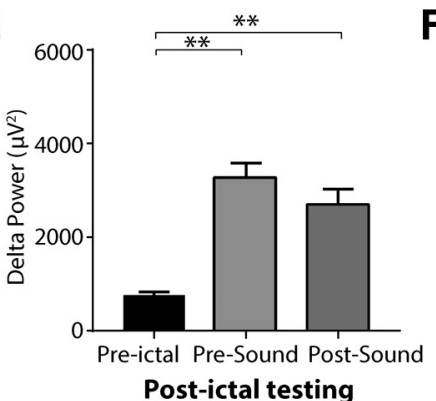

$\mathbf{F}$

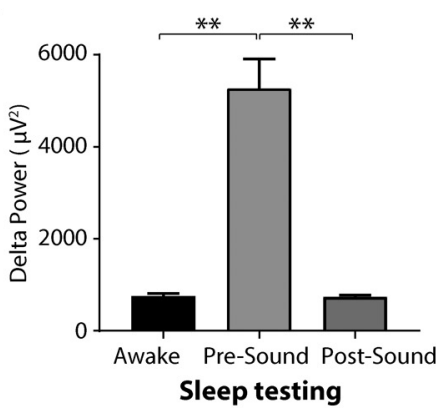

Figure 4. Responses to auditory stimuli are impaired in the lever-press $E / A$ task during the postictal period but are preserved during naturally occurring sleep. $A$, Example of lever-press $E / A$ task during the postictal period. The auditory signal failed to eliminate cortical slow waves, and the rat failed to avoid the shock (pushed lever only after shock delivery). $\boldsymbol{B}$, In naturally occurring sleep, the auditory stimulus abolished cortical slowing and woke up rat to press lever and avoid shock. $C$, Avoidance percentage for E/A task is decreased during postictal period when compared with natural sleep, which is comparable to that of animals in the awake state. $\boldsymbol{D}$, Response time in E/A task is longer during postictal period when compared with response time in natural sleep and awake state. $\boldsymbol{E}$, The auditory stimulus does not abolish postictal delta slow wave activity in comparison to the preictal period. $\boldsymbol{F}$, In contrast the auditory stimulus markedly reduces delta slowing during natural sleep; $n=9$ rats, 49 natural sleep sessions and 23 postictal sessions. All results are mean $\pm S E M ; * * p<0.01$. Ctx, orbital frontal cortex.

cortical LFP; (2) during lever-press E/A tasks, trials in which based on video review the rat touched the lever accidentally with head stage instead of purposefully with paw.

Group analyses were performed by first pooling all results within each animal by averaging, and statistics were then calculated across animals $(n=$ number of animals). This approach is more conservative because it yields a lower sample size than performing statistics across trials ( $n=$ number of trials). However, because pooling data within animals could potentially introduce bias (because of animals with outlier values and low number of trials), we repeated all analyses by calculating statistics across trials without pooling, which yielded results essentially identical to those reported here (data not shown). Results are reported as mean \pm SEM. A two-tailed unpaired Student's $t$ test was used to compare two groups. A one-way ANOVA followed by post hoc Tukey's test was used to compare three or more groups. Differences with $p<0.05$ were considered statistically significant. All statistical analyses were done using GraphPad Prism 7 software.

\section{Results}

Intralaminar thalamic stimulation produces modest improvement in spontaneous lick task

Rats were successfully trained to perform the sucrose reward spontaneous licking task (Fig. $1 A, B$ ) receiving $\geq 90 \%$ of rewards in an average of $4.7 \pm 0.2$ daily training sessions before surgery (range 4-6d). During training, the licking rate increased progressively day by day on average, with a rate of $2.1 \pm 0.6$ licks/s for the first session (mean \pm SEM), $2.8 \pm 0.6$ for the second day, $3.4 \pm 0.6$ for the third day, and $3.7 \pm 0.6$ for the fourth. After surgery, it took 3.5 daily sessions on average to retrain rats to receive $\geq 90 \%$ of rewards and to lick the spout nearly continuously while in the testing chamber. This procedure provided a good behavioral baseline to study changes during the ictal and postictal periods, as well as the effects of thalamic CL DBS.

In sucrose reward experiments performed in chronically implanted awake animals during the ictal period, poly-spike discharges in the frontal cortical and HC LFP were observed in both thalamic CL DBS and sham groups (Fig. 2). After seizure termination, postictal cortical slow waves were observed in the orbital frontal cortex (Fig. 2B) as described previously in this rodent model as well as in human patients (Blumenfeld et al., 2004; Englot et al., 2008, 2010; Gummadavelli et al., 2015a). In the DBS group, thalamic CL stimulation abolished postictal slow waves in the cortical LFP (Fig. 2C) unlike sham DBS controls (Fig. $2 B$ ). Group time course analysis of the cortical delta band power $(n=12$ rats, sham sessions $=55$, thalamic CL DBS sessions $=55$ ) demonstrated significantly decreased low-frequency power during the postictal 1 - to 250 -s $(p=0.0039)$ and 
A

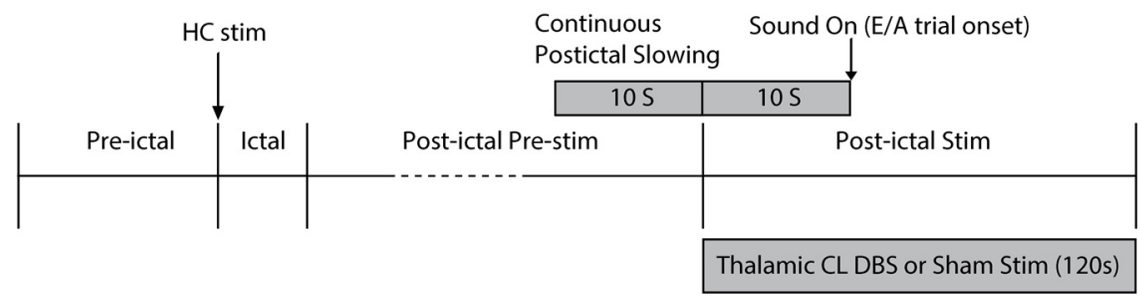

B

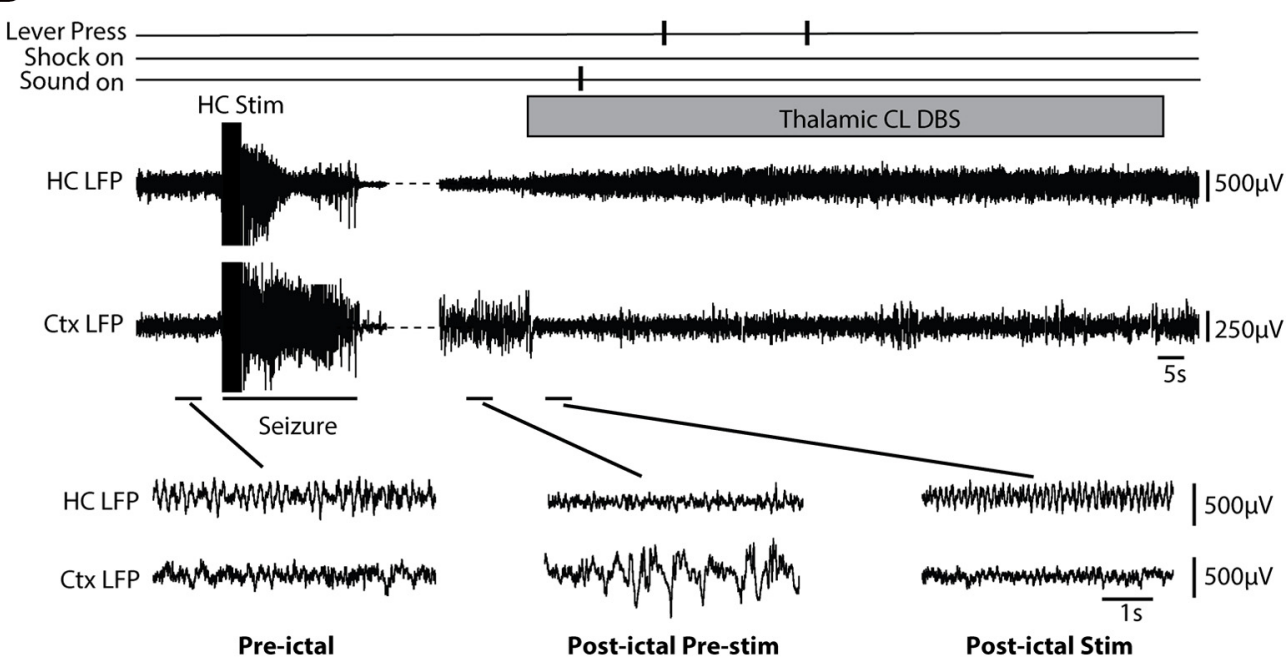

Figure 5. Bilateral CL stimulation produces cortical physiological arousal and markedly improves postictal lever-press E/A task performance. $A$, Experimental protocol and sequence of preictal, ictal, postictal pre-stim, and postictal-stim epochs. HC stimulation for $2 \mathrm{~s}$ at $60 \mathrm{~Hz}$ induces a secondarily generalized seizure. Following the occurrence of 10-s continuous postictal cortical slowing, bilateral thalamic CL DBS or sham control DBS is initiated and continued for $2 \mathrm{~min}(120 \mathrm{~s})$. A new E/A trial is initiated $10 \mathrm{~s}$ after thalamic stimulation onset to test response to sound. $\boldsymbol{B}$, Example of $\mathrm{CL}$ stimulation in the postictal period after a secondarily generalized seizure in the lever-press $\mathrm{E} / \mathrm{A}$ task. A 95 -s break in the recording is indicated with interrupted line to show postictal cortical slow-wave activity just before $\mathrm{CL}$ stimulation. Following initiation of $\mathrm{CL}$ stimulation cortical slow waves are reduced and the animal responds appropriately by lever press to the sound stimulus. Magnified insets show the indicated preictal, postictal pre-stim, and postictal stim epochs. Cortical slow waves seen in the postictal pre-stim epoch are dramatically reduced during postictal stim epoch. Cortical LFP during the Postictal Stim epoch resembles the baseline preictal epoch. Ctx, orbital frontal cortex.

postictal 250- to 500 -s $(p=0.0302)$ periods in the thalamic CL DBS group compared with the sham group (Fig. $3 A, B$ ). The seizure durations of the two groups showed no statistical difference (DBS $71.3 \pm 3.3 \mathrm{~s}$ vs sham $75.1 \pm 2.1 \mathrm{~s}$, mean $\pm \mathrm{SEM}, p=$ 0.3329).

Behaviorally, both groups had abrupt interruption of licking during the ictal period (Fig. 3C). During the postictal period, there was a gradual improvement in licking which tended to be slightly faster in the thalamic CL DBS group; however, this did not reach statistical significance (Fig. $3 C, D$ ). In the post-stim period, a statistically significant difference in the licking rate emerged between DBS and sham groups during both the poststim 1 - to 250-s $(p=0.0399)$ and post-stim 250 - to $500-\mathrm{s}$ $(p=0.0048)$ periods (Fig. $3 C, D)$. Thus, although thalamic CL DBS produced a clear improvement in cortical physiological arousal evidenced by reduced delta frequency activity in the postictal period, improvement in a self-motivated behavior such as spontaneous licking was only modest and did not reach statistical significance until long after seizures had terminated. These results encouraged us to further investigate behavior by using a task with stronger external motivating factors, which could further evaluate ability to respond.

Lever-press E/A performance is impaired postictally but spared in natural occurring sleep

Rats were successfully trained to avoid shock on $\geq 90 \%$ of trials during the E/A task (Fig. $1 A, C$ ) after an average of $7.4 \pm 0.4$ daily sessions (range 6-10 d) before surgery. The avoidance percentage in training sessions progressively increased from $77.9 \pm 4.3 \%$ (first session) to $98.3 \pm 1.1 \%$ (seventh session), and the response time decreased from $21.5 \pm 1.3 \mathrm{~s}$ (first session) to $6.7 \pm 0.6 \mathrm{~s}$ (seventh session). After surgery, retraining to $\geq 90 \%$ avoidance on the E/A task took an average of $1.4 \pm 0.2 \mathrm{~d}$.

Although cortical slow waves in the postictal state were similar to those observed in the frontal cortex when the animal was naturally sleeping, the level of responsiveness was different in these two conditions. During the lever-press E/A task, an auditory stimulus was used as the warning signal, which allowed us to identify behavioral differences between the postictal period and naturally occurring sleep. This auditory stimulus failed to elicit behavioral or electrophysiological arousal in the postictal state but was sufficient during natural sleep to wake up rats and abolish sleep-related cortical slow waves. The cortical slow waves persisted after seizures, and rats usually could not achieve avoidance during the E/A task (Fig. $4 A$ ). In contrast, during natural sleep the sound stimulus often elicited a cortical physiological arousal and behavioral response by lever press (Fig. 4B). Group analysis indicated a significant difference in behavioral responses between the postictal state and naturally occurring sleep (Fig. 4C, $D)$. During the postictal period, avoidance percentage was $6.5 \pm 4.3 \%$ and response time was $65.0 \pm 3.1 \mathrm{~s}$ ( $n=9$ rats; 23 postictal sessions). During naturally occurring sleep, avoidance percentage was $93.1 \pm 4.6 \%$ and response time was $15.1 \pm 3.7 \mathrm{~s}$ ( $n=9$ rats; 49 sleep sessions). In addition, the 0 - to $4-\mathrm{Hz}$ deltawave power did not change significantly in response to the sound stimulus during the postictal period $\left(3327 \pm 368.2 \mu \mathrm{V}^{2}\right.$ presound vs $2807 \pm 410.4 \mu \mathrm{V}^{2}$ postsound), whereas delta power decreased 
A

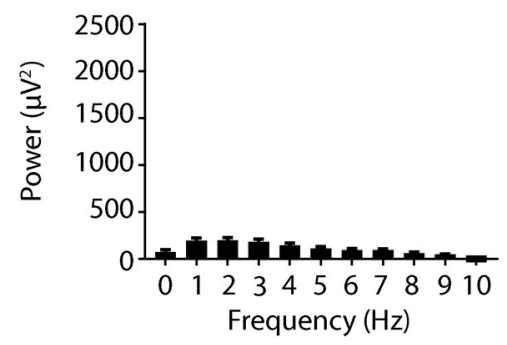

B

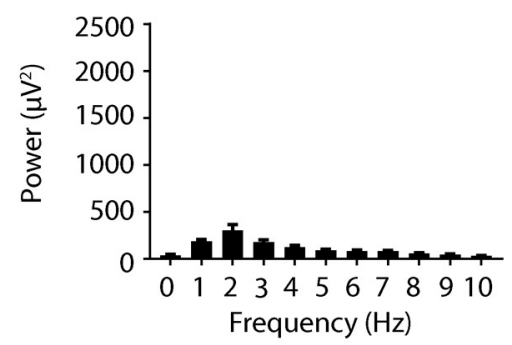

Sham Post-ictal Pre-stim

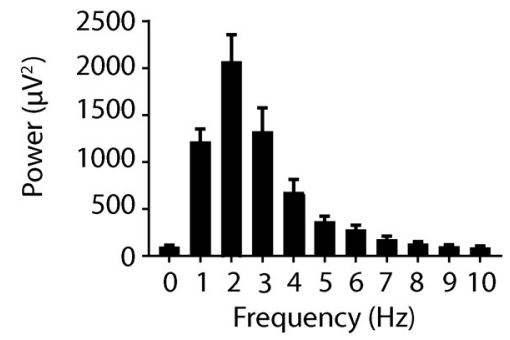

DBS Post-ictal Pre-stim

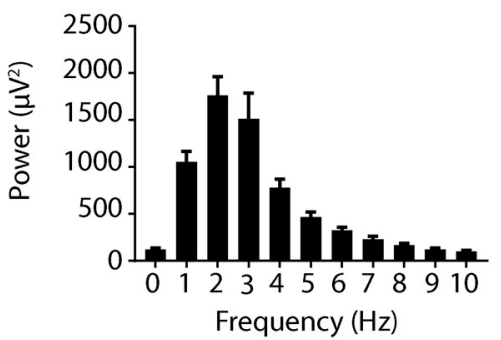

Sham Post-ictal Stim

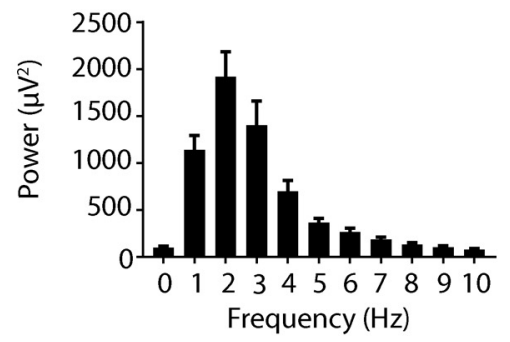

DBS Post-ictal Stim

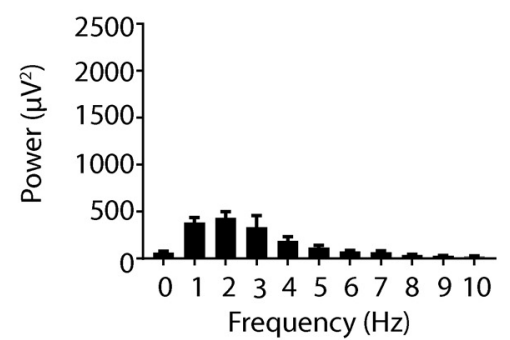

C

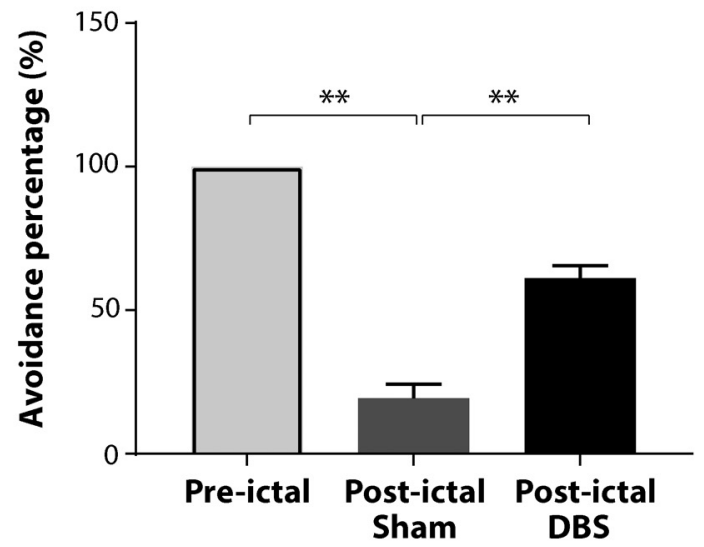

D

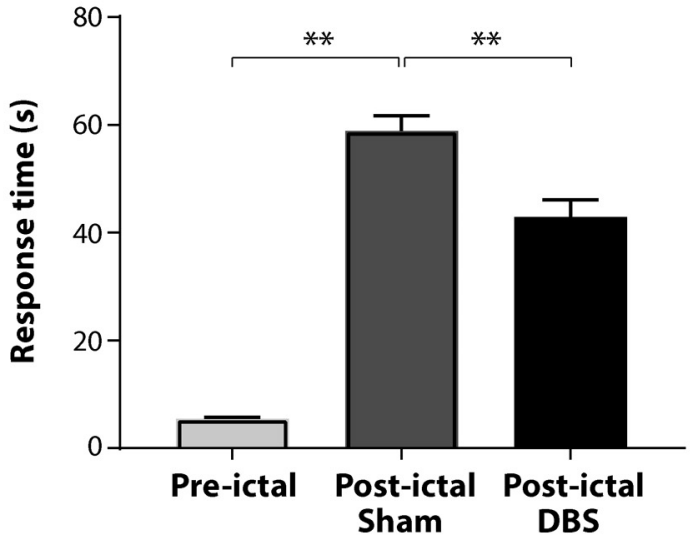

Figure 6. Thalamic CL stimulation improves cortical physiological arousal and lever-press E/A task performance during the postictal period. $A, B$, Group analysis of cortical LFP power shows that postictal thalamic CL DBS decreases low-frequency power during the postictal stim epoch compared with sham control group. C, Significant increases in avoidance percentage for E/A task are observed in postictal DBS group compared with sham control group. $D$, Decreased response time for E/A task is also observed in the DBS group when compared with sham group; $n=9$ rats, 47 sham sessions and $51 \mathrm{DBS}$ sessions. All results are mean $\pm \mathrm{SEM} ;{ }^{* *} p<0.01$. Ctx, orbital frontal cortex.

significantly after sound onset during naturally occurring sleep (5157 $\pm 910.5 \mu \mathrm{V}^{2}$ presound vs $729.5 \pm 66.32 \mu \mathrm{V}^{2}$ postsound; Fig. $3 E, F)$. The lever-press E/A task therefore provides a paradigm with a potent external stimulus where rats are capable of responding even during natural sleep but are highly impaired postictally, modeling impaired responses to hazardous situations encountered by epilepsy patients in the postictal period.

Intralaminar thalamic stimulation markedly improves postictal lever-press E/A task performance

We next tested the effect of thalamic CL stimulation in the leverpress E/A task during the postictal period. CL stimulation abolished postictal slow waves in the cortical LFP and restored lever-press responses to the sound stimulus (Fig. 5). Group analysis showed that CL stimulation decreased low-frequency power during the postictal period (mean 0 - to $4-\mathrm{Hz}$ delta-wave power $1059.75 \pm 151.52 \mu \mathrm{V}^{2}$ CL DBS postictal pre-stim vs $285.59 \pm$ $72.47 \mu \mathrm{V}^{2} \mathrm{CL}$ DBS postictal stim, $p=0.0003$; Fig. $6 B$ ), whereas sham stimulation did not change low-frequency power [mean 0to $4-\mathrm{Hz}$ delta-wave power $1030.33 \pm 195.39 \mu \mathrm{V}^{2}$ sham postictal pre-stim vs $979.56 \pm 184.48 \mu \mathrm{V}^{2}$ sham postictal stim, $p=0.4283$ (Fig. $6 A$ ); $n=9$ rats; 51 DBS trials, 47 sham trials (Fig. $6 A, B$ )]. In addition, CL stimulation markedly improved lever-press E/A task performance. Rats in the DBS group achieved higher avoidance percentage than those in the sham group $(66.1 \pm 4.2 \%$ for DBS vs $17.8 \pm 5.2 \%$ for sham; $p<0.0001$; Fig. $6 C$ ), and the response time was shorter in DBS group than that in sham group $(42.5 \pm 2.3 \mathrm{~s}$ for DBS vs $59.9 \pm 2.3 \mathrm{~s}$ for sham; $p<0.0001$; Fig. 6D).

Because we observed a positive effect of thalamic CL DBS on the E/A task in the postictal period, we wanted to ensure the observed effects were not simply because of differences in seizure magnitude or timing in the DBS versus sham groups. We therefore compared the DBS versus sham group data for seizure duration, root mean square (Vrms) seizure amplitude in the hippocampal and cortical channels, as well as E/A stimulus onset delay time from seizure end. No significant differences between DBS and sham groups were detected. Mean seizure duration was $81.8 \pm 3.1 \mathrm{~s}$ for DBS versus $75.8 \pm 3.5 \mathrm{~s}$ for $\operatorname{sham}(p=0.20)$; seizure Vrms amplitude in the hippocampal channel was $95.3 \pm 9.4 \mathrm{mV}$ for DBS versus $82.1 \pm 9.7 \mathrm{mV}$ for $\operatorname{sham}(p=0.34)$; 
seizure Vrms in the cortical channel was $133.0 \pm 7.4 \mathrm{mV}$ for DBS versus $147.5 \pm 8.3 \mathrm{mV}$ for sham $(p=0.20)$; E/A sound stimulation onset time from seizure end was $159.7 \pm 14.3 \mathrm{~s}$ for DBS versus $131.0 \pm 11.0 \mathrm{~s}$ for sham ( $p=0.56 ; n=9$ rats, 51 seizures with CL DBS, 47 with sham).

\section{Discussion}

In this study, we developed two behavioral tasks to evaluate postictal behavior in a rodent model of secondarily generalized limbic seizures. By combining these behavioral tests with electrophysiological recordings, we found that the postictal period was characterized by frontal cortical slow waves and impaired behavior on both tasks. High-frequency bilateral intralaminar thalamic stimulation of the thalamic CL nucleus increased postictal cortical physiological arousal, producing limited improvement in behavior on the sucrose reward task and marked improvement on the lever-press shock escape-avoidance task. These results suggest a novel therapeutic strategy which may be translated to help restore postictal arousal following secondarily generalized seizures in patients with medically and surgically refractory epilepsy. A treatment of this kind would be especially beneficial if also coupled with improved arousal during the ictal period, at least for focal seizures, in the same individuals (Gummadavelli et al., 2015b; Kundishora et al., 2017).

The postictal state has been well characterized in patients clinically and electrophysiologically (Rémi and Noachtar, 2010; Farrell et al., 2017; Peng et al., 2017). However, to our knowledge, very limited work has been done to investigate postictal behavioral changes in rodent models. The ability to evaluate postictal behavioral impairment in rodents provides an important approach for testing novel therapeutic strategies with high clinical translational relevance. We developed two behavioral tasks to pursue this goal. In the sucrose reward spontaneous licking task, the increase in delta wave cortical LFP power and decrease in licking rate demonstrated that during the postictal period, there is impaired cortical electrophysiological arousal with corresponding behavioral deficits. These findings were further confirmed in the E/A task, in which the rats showed impaired response to an auditory stimulus during the postictal period even when compared with naturally occurring sleep. It has been well established that some auditory processing and behavioral responses can occur during sleep (Oswald et al., 1960; Halperin and Iorio, 1981). However, the present results suggest that depressed arousal is more severe during the postictal state, in agreement with recent human work showing fundamental physiological

differences between natural sleep and the postictal state (Lundstrom et al., 2019). Postictal physiological and behavioral level of consciousness thus appears markedly impaired in rats, and most importantly, the availability of this rodent model makes it possible to evaluate the effects of therapeutic strategies.

The central thalamus receives arousal-related inputs from the brainstem (Bayer et al., 2002; Krout et al., 2002) and is hypothesized to support synchronization and effort regulation in neural networks that underlie cognitive functions (Jones, 2009). It has long been postulated that the thalamus works as a major network hub in ictal and postictal states of impaired consciousness (Penfield, 1936). Previous studies in chronically implanted animals demonstrated that CL stimulation induced cortical desynchronization in LFP recordings during the postictal state after secondarily generalized seizures (Gummadavelli et al., 2015a). In addition, Kundishora et al. found that dual-site stimulation of the CL and pontine nucleus oralis (PnO) bilaterally restored an awake-appearing cortical physiological state and improved behavioral arousal during the ictal period in focal seizures (Kundishora et al., 2017). These studies emphasize the important role of $\mathrm{CL}$ in maintaining consciousness. However, consciousness level in these studies was evaluated based on simple observation of resumed exploratory behavior and movement in rats. Evaluation of task performance requiring purposeful behavior was needed beyond these more basic previous behavioral observations to further explore the efficacy and safety of CL stimulation during the postictal period. The present study focused on evaluation of task performance based on purposeful behaviors in the postictal period after secondarily generalized seizures. Because this is a state in which patients are typically profoundly impaired this work has important clinical relevance.

Focal seizures also often impair consciousness ictally and postictally, although to a lesser extent than generalized seizures (McPherson et al., 2012). Focal seizures should be investigated with purposeful behavioral tasks as well in future work with rodent models. In addition, to further investigate potential side effects and benefits in a model more closely resembling treatment of epilepsy patients, the effects of CL stimulation following closed-loop seizure detection in spontaneous seizure rodent models should also be done. Another subject for further investigation is potential direct effects of CL stimulation on seizure severity. In our experiments with the sucrose reward task, CL stimulation began immediately with seizure onset (Fig. 2A), thus fully overlapping the seizure period. We did not see a significant difference in seizure duration or amplitude (based on delta power; Fig. $3 A, B)$ during the ictal period, suggesting the effects of CL stimulation was not simply to reduce seizure severity. In addition, we did see benefits of CL stimulation even when it was delivered fully in the postictal period with no overlap of the seizure time period (Fig. 5A,B), producing improvements in both postictal physiology and behavior on the E/A task (Fig. 6). Nevertheless, additional studies of possible direct effects of CL stimulation on seizure severity would be valuable.

In the current study, rats in the group that received CL stimulation showed modest improvement in performance during the postictal period in the sucrose reward task and marked improvement on the E/A task. Interestingly, in the sucrose reward task, electrophysiological improvement (decreased delta-wave power) was obvious during the postictal DBS period; however, significant behavioral improvement (increased licking rate) was observed only at later times even after DBS had ended. In other words, the behavioral licking response seemed to lag behind the electrophysiologic changes. These findings suggest that during the postictal period, when DBS abolished cortical slow waves, other factors aside from cortical arousal might have contributed to the persistently reduced spontaneous licking responses. In contrast, with the shock E/A task postictal thalamic CL DBS dramatically improved lever-press responses. Of note, the sucrose reward task we developed was a positive reinforcement type task; rats received no punishment if they did not lick and received rewards when they did. Thus, we can speculate that the motivation to perform in this task was less than in the E/A task, where failing to perform led to a negative consequence. In addition, the absence of an auditory stimulus as a cue to signal sucrose availability would require more internal motivation for rats to initiate performance compared with the E/A task where an auditory warning signaled incipient arrival of the aversive shock. Compared with the sucrose reward task, the E/A task we developed was a negative reinforcement type task. The rats received 
an aversive stimulus in the form of an electrical shock if they failed to push the lever after the auditory signal. Our results demonstrated that avoidance percentage for the rats that received CL DBS was significantly higher and response times significantly shorter when compared with the sham group. Importantly, these findings suggest that thalamic CL neurostimulation could have a potential therapeutic benefit in promoting self-preservation and thus improving the safety of patients with epilepsy in the postictal period.

In conclusion, we have developed two behavioral tasks that allowed us to characterize the postictal state in a rodent model of secondarily generalized limbic seizures. CL DBS decreased cortical slow wave activity and improved task performance in the postictal period, particularly in the E/A task. Because preclinical task performance studies are crucial to explore the effectiveness and safety of DBS treatment, our work is clinically relevant as it could support and help set the foundations for human neurostimulation trials (https://braininitiative.nih.gov/funded-awards/ thalamic-stimulation-prevent-impaired-consciousness-epilepsy). In particular, therapeutic benefits of DBS during the ictal period could be substantially augmented by improved responsiveness in the postictal period in patients with medically and surgically refractory epilepsy.

\section{References}

Baker JL, Ryou JW, Wei XF, Butson CR, Schiff ND, Purpura KP (2016) Robust modulation of arousal regulation, performance, and frontostriatal activity through central thalamic deep brain stimulation in healthy nonhuman primates. J Neurophysiol 116:2383-2404.

Bayer L, Eggermann E, Saint-Mleux B, Machard D, Jones BE, Mühlethaler M, Serafin M (2002) Selective action of orexin (hypocretin) on nonspecific thalamocortical projection neurons. J Neurosci 22:7835-7839.

Benedetti-Isaac JC, Torres-Zambrano M, Vargas-Toscano A, Perea-Castro E, Alcalá-Cerra G, Furlanetti LL, Reithmeier T, Tierney TS, Anastasopoulos C, Fonoff ET, Contreras Lopez WO (2015) Seizure frequency reduction after posteromedial hypothalamus deep brain stimulation in drug-resistant epilepsy associated with intractable aggressive behavior. Epilepsia $56: 1152-1161$

Blumenfeld H (2012) Impaired consciousness in epilepsy. Lancet Neurol 11:814-826.

Blumenfeld H, Rivera M, McNally KA, Davis K, Spencer DD, Spencer SS (2004) Ictal neocortical slowing in temporal lobe epilepsy. Neurology 63:1015-1021.

Carlsson G, Wiegand G, Stephani U (2011) Interictal and postictal performances on dichotic listening test in children with focal epilepsy. Brain Cogn 76:310-315.

Elliott IM, Lach L, Smith ML (2005) I just want to be normal: a qualitative study exploring how children and adolescents view the impact of intractable epilepsy on their quality of life. Epilepsy Behav 7:664-678.

Englot DJ, Mishra AM, Mansuripur PK, Herman P, Hyder F, Blumenfeld H (2008) Remote effects of focal hippocampal seizures on the rat neocortex. J Neurosci 28:9066-9081.

Englot DJ, Yang L, Hamid H, Danielson N, Bai X, Marfeo A, Yu L, Gordon A, Purcaro MJ, Motelow JE, Agarwal R, Ellens DJ, Golomb JD, Shamy MCF, Zhang H, Carlson C, Doyle W, Devinsky O, Vives K, Spencer DD, et al. (2010) Impaired consciousness in temporal lobe seizures: role of cortical slow activity. Brain 133:3764-3777.

Farrell JS, Colangeli R, Wolff MD, Wall AK, Phillips TJ, George A, Federico P, Teskey GC (2017) Postictal hypoperfusion/hypoxia provides the foundation for a unified theory of seizure-induced brain abnormalities and behavioral dysfunction. Epilepsia 58:1493-1501.

Feng L, Motelow JE, Ma C, Biche W, McCafferty C, Smith N, Liu M, Zhan Q, Jia R, Xiao B, Duque A, Blumenfeld H (2017) Seizures and sleep in the thalamus: focal limbic seizures show divergent activity patterns in different thalamic nuclei. J Neurosci 37:11441-11454.

Fisher RS, Schachter SC (2000) The postictal state: a neglected entity in the management of epilepsy. Epilepsy Behav 1:52-59.
Fisher RS, Velasco AL (2014) Electrical brain stimulation for epilepsy. Nat Rev Neurol 10:261-270.

Gummadavelli A, Motelow JE, Smith N, Zhan Q, Schiff ND, Blumenfeld H (2015a) Thalamic stimulation to improve level of consciousness after seizures: evaluation of electrophysiology and behavior. Epilepsia 56:114124.

Gummadavelli A, Kundishora AJ, Willie JT, Andrews JP, Gerrard JL, Spencer DD, Blumenfeld H (2015b) Improving level of consciousness in epilepsy with neurostimulation. Neurosurg Focus 38:EX.

Halperin JM, Iorio LC (1981) Responsitivity of rats to neutral and dangersignaling stimuli during sleep. Behav Neural Biol 33:213-219.

Heck CN, King-Stephens D, Massey AD, Nair DR, Jobst BC, Barkley GL, Salanova V, Cole AJ, Smith MC, Gwinn RP, Skidmore C, Van Ness PC, Bergey GK, Park YD, Miller I, Geller E, Rutecki PA, Zimmerman R, Spencer DC, Goldman A, et al. (2014) Two-year seizure reduction in adults with medically intractable partial onset epilepsy treated with responsive neurostimulation: final results of the RNS system pivotal trial. Epilepsia 55:432-441.

Helmstaedter C, Elger CE, Lendt M (1994) Postictal courses of cognitive deficits in focal epilepsies. Epilepsia 35:1073-1078.

Hesdorffer DC, Tomson T (2013) Sudden unexpected death in epilepsy. Potential role of antiepileptic drugs. CNS Drugs 27:113-119.

Jones EG (2009) Synchrony in the interconnected circuitry of the thalamus and cerebral cortex. Ann NY Acad Sci 1157:10-23.

Josephson CB, Engbers JD, Sajobi TT, Jette N, Agha-Khani Y, Federico P, Murphy W, Pillay N, Wiebe S (2016) An investigation into the psychosocial effects of the postictal state. Neurology 86:723-730.

Klinger N, Mittal S (2018) Deep brain stimulation for seizure control in drug-resistant epilepsy. Neurosurg Focus 45:E4.

Krout KE, Belzer RE, Loewy AD (2002) Brainstem projections to midline and intralaminar thalamic nuclei of the rat. J Comp Neurol 448:53-101.

Kundishora AJ, Gummadavelli A, Ma C, Liu M, McCafferty C, Schiff ND, Willie JT, Gross RE, Gerrard J, Blumenfeld H (2017) Restoring conscious arousal during focal limbic seizures with deep brain stimulation. Cereb Cortex 27:1964-1975.

Kuo J, Zhao W, Li CS, Kennedy JD, Seyal M (2016) Postictal immobility and generalized EEG suppression are associated with the severity of respiratory dysfunction. Epilepsia 57:412-417.

Liu J, Lee HJ, Weitz AJ, Fang Z, Lin P, Choy M, Fisher R, Pinskiy V, Tolpygo A, Mitra P, Schiff N, Lee JH (2015) Frequency-selective control of cortical and subcortical networks by central thalamus. Elife 4:e09215.

Lundstrom BN, Boly M, Duckrow R, Zaveri HP, Blumenfeld H (2019) Slowing less than $1 \mathrm{~Hz}$ is decreased near the seizure onset zone. Sci Rep 9:6218.

MacEachern SJ, D'Alfonso S, McDonald RJ, Thornton N, Forkert ND, Buchhalter JR (2017) Most children with epilepsy experience postictal phenomena, often preventing a return to normal activities of childhood. Pediatr Neurol 72:42-50.e3.

McPherson A, Rojas L, Bauerschmidt A, Ezeani CC, Yang L, Motelow JE, Farooque P, Detyniecki K, Giacino JT, Blumenfeld H (2012) Testing for minimal consciousness in complex partial and generalized tonic-clonic seizures. Epilepsia 53:e180-e183.

Motelow JE, Li W, Zhan Q, Mishra AM, Sachdev RN, Liu G, Gummadavelli A, Zayyad Z, Lee HS, Chu V, Andrews JP, Englot DJ, Herman P, Sanganahalli BG, Hyder F, Blumenfeld H (2015) Decreased subcortical cholinergic arousal in focal seizures. Neuron 85:561-572.

Norden AD, Blumenfeld H (2002) The role of subcortical structures in human epilepsy. Epilepsy Behav 3:219-231.

Oswald I, Taylor AM, Treisman M (1960) Discriminative responses to stimulation during human sleep. Brain 83:440-453.

Paxinos G, Waston C (1998) The rat brain in stereotaxic coordinates. San Diego: Academic Press.

Penfield W (1936) Epilepsy and surgical therapy. Arch NeurPsych 36:449484.

Peng W, Danison JL, Seyal M (2017) Postictal generalized EEG suppression and respiratory dysfunction following generalized tonic-clonic seizures in sleep and wakefulness. Epilepsia 58:1409-1414.

Redinbaugh MJ, Phillips JM, Kambi NA, Mohanta S, Andryk S, Dooley GL, Afrasiabi M, Raz A, Saalmann YB (2020) Thalamus modulates consciousness via layer-specific control of cortex. Neuron 106:66-75.e2.

Rémi J, Noachtar S (2010) Clinical features of the postictal state: correlation with seizure variables. Epilepsy Behav 19:114-117. 
Rugg-Gunn F, Duncan J, Hjalgrim H, Seyal M, Bateman L (2016) From unwitnessed fatality to witnessed rescue: nonpharmacologic interventions in sudden unexpected death in epilepsy. Epilepsia 57 [Suppl 1]:26-34.

Ryvlin P, Nashef L, Lhatoo SD, Bateman LM, Bird J, Bleasel A, Boon P, Crespel A, Dworetzky BA, Høgenhaven H, Lerche H, Maillard L, Malter MP, Marchal C, Murthy JM, Nitsche M, Pataraia E, Rabben T, Rheims S, Sadzot B, et al. (2013) Incidence and mechanisms of cardiorespiratory arrests in epilepsy monitoring units (MORTEMUS): a retrospective study. Lancet Neurol 12:966-977.

Schiff ND, Giacino JT, Kalmar K, Victor JD, Baker K, Gerber M, Fritz B, Eisenberg B, Biondi T, O’Connor J, Kobylarz EJ, Farris S, Machado A, McCagg C, Plum F, Fins JJ, Rezai AR (2007) Behavioural improvements with thalamic stimulation after severe traumatic brain injury. Nature 448:600-603.

Schiff ND, Shah SA, Hudson AE, Nauvel T, Kalik SF, Purpura KP (2013) Gating of attentional effort through the central thalamus. J Neurophysiol 109:1152-1163.

Schmidt D, Noachtar S (2010) Outlook: the postictal state-future directions for research. Epilepsy Behav 19:191-192.

Servatius RJ, Jiao X, Beck KD, Pang KC, Minor TR (2008) Rapid avoidance acquisition in Wistar-Kyoto rats. Behav Brain Res 192:191-197.
Seyal M, Bateman LM, Li CS (2013) Impact of periictal interventions on respiratory dysfunction, postictal EEG suppression, and postictal immobility. Epilepsia 54:377-382.

Theodore WH (2010) The postictal state: effects of age and underlying brain dysfunction. Epilepsy Behav 19:118-120.

Todd RB (2005) The Lumleian Lectures for 1849. On the pathology and treatment of convulsive diseases. Epilepsia 46:995-1009.

Tomson T, Surges R, Delamont R, Haywood S, Hesdorffer DC (2016) Who to target in sudden unexpected death in epilepsy prevention and how? Risk factors, biomarkers, and intervention study designs. Epilepsia 57 [Suppl 1]:4-16.

Van der Werf YD, Witter MP, Groenewegen HJ (2002) The intralaminar and midline nuclei of the thalamus. Anatomical and functional evidence for participation in processes of arousal and awareness. Brain Res Brain Res Rev 39:107-140.

Werhahn KJ (2010) Weakness and focal sensory deficits in the postictal state. Epilepsy Behav 19:138-139.

Zhan Q, Buchanan GF, Motelow JE, Andrews J, Vitkovskiy P, Chen WC, Serout F, Gummadavelli A, Kundishora A, Furman M, Li W, Bo X, Richerson GB, Blumenfeld H (2016) Impaired serotonergic brainstem function during and after seizures. J Neurosci 36:2711-2722. 